\title{
Effect of TLR2 monoclonal antibody on new blood vessels and immune rejection response after keratoplasty
}

\author{
Wang Ji, Yuan Jianshu, Wen Feng * \\ Department of Ophthalmology, Ningbo Eye Hospital, Ningbo 315040, China \\ *For correspondence: Email: fw1280@163.com
}

Sent for review: 11 October 2018

Revised accepted: 28 December 2018

\begin{abstract}
Purpose: To investigate the effect of TLR2 (toll-like receptor 2) monoclonal antibody on formation of new blood vessels and immune rejection response after keratoplasty.

Methods: The rats were randomly divided into negative control group consisting of 16 rats from allogeneic corneal transplantation, and TLR2 monoclonal antibody group (study group) made up of 16 rats from allogeneic corneal transplantation treated with TLR2 monoclonal antibody. A group of 8 rats served as normal control. The study group was treated with $0.5 \mathrm{~g} \cdot \mathrm{L}-1$ TLR2 monoclonal antibody through sub-conjunctival injection once daily for 5 days, while the normal control and negative control groups were given an equivalent volume of normal saline in place of TLR2. Corneal transparency and neovascularization were observed under slit lamp daily after operation, and scored using rejection index.

Results: In the TLR2 monoclonal antibody group, the corneal structure was still clear, and only a few inflammatory cells infiltrated the stromal layer. There were trace amounts of TLR2 expression in the corneal epithelium of rats in normal control group, negative control group and TLR2 monoclonal antibody group. In the negative control group, the expression of TLR2 in the corneal epithelium and stromal cells significantly increased, especially in the stromal layer.

Conclusion: TLR2 monoclonal antibody exerts a significant effect on neovascularization and immune rejection after corneal transplantation in rats. Thus, it may be clinically suitable for the prevention and treatment of rejection arising from corneal transplantation.
\end{abstract}

Keywords: TLR2 monoclonal antibody, Corneal transplantation, Neovascularization, Immune rejection

\begin{abstract}
This is an Open Access article that uses a funding model which does not charge readers or their institutions for access and distributed under the terms of the Creative Commons Attribution License (http://creativecommons.org/licenses/by/4.0) and the Budapest Open Access Initiative (http://www.budapestopenaccessinitiative.org/read), which permit unrestricted use, distribution, and reproduction in any medium, provided the original work is properly credited.
\end{abstract}

Tropical Journal of Pharmaceutical Research is indexed by Science Citation Index (SciSearch), Scopus, International Pharmaceutical Abstract, Chemical Abstracts, Embase, Index Copernicus, EBSCO, African Index Medicus, JournalSeek, Journal Citation Reports/Science Edition, Directory of Open Access Journals (DOAJ), African Journal Online, Bioline International, Open-J-Gate and Pharmacy Abstracts

\section{INTRODUCTION}

Keratopathy is the second leading cause of blindness after cataracts [1]. A significant number of keratopathy patients can be cured only through corneal transplantation. The relative immunity "state of pardon" of corneal tissue makes the success rate of corneal transplantation very high $[2,3]$. However, as the number of corneal transplant patients increases yearly, the cumulative graft rejection cannot be ignored [4]. In particular, the incidence of postoperative rejection can be as high as $65 \%$ especially when there is high degree of neovascularization due to chronic corneal inflammation [5]. 
Toll-like receptor (TLR) is a bridge linking innate immunity and acquired immunity [6]. It is the most widely expressed molecule in the cloned human TLR family, which recognizes most pathogenic microorganisms. In previous experiments, it was confirmed at the gene, molecular and cellular levels that the expression of TLR2 mRNA was significantly increased and transferred to the cell membrane on the $9^{\text {th }}$ day after corneal allograft transplantation in rats [7]. In the present study, the effect of TLR2 monoclonal antibody on neovascularization and immune rejection after corneal transplantation was investigated.

\section{EXPERIMENTAL}

\section{Materials}

TLR2 monoclonal antibody was from Biolegend Company (USA); TLR2 primary antibody was from Proteintech Company (USA), while antiGoat Ig was from DaKo (Denmark). Forty female Wistar rats (SPF grade), each weighing about $220 \mathrm{~g}$, and 16 female Sprague Dawley (SD) rats, with body weight of about $220 \mathrm{~g} / \mathrm{rat}$ were raised and provided by the Institute of Model Animals of Nanjing University.

Sixteen SD rats were used as donors, while 32 Wistar rats served as recipients. Allogeneic penetrating keratoplasty model was established [7] in the 32 rats which were subsequently randomly divided into two groups: negative control group and TLR2 monoclonal antibody group, each containing 16 rats. A group of 8 female Wistar rats (SPF grade) were used as the normal control group. This research was approved by the Animal Ethical Committee of Ningbo Eye Hospital (approval no. 20178261), and conducted according to the guidelines of "Principles of Laboratory Animal Care" (NIH publication no. 85-23, revised 1985) [8].

\section{Establishment of rat model of allogeneic penetrating corneal transplantation}

A rat model of allogeneic penetrating corneal transplantation was established according to a previous report [7]. Pentobarbital sodium (3\%, $1.5 \mathrm{~mL} / \mathrm{kg}$ ) was injected intraperitoneally to the rats. Before operation, topiricamide was used twice to fully dilate the pupil. Tetracaine hydrochloride served as topical anesthesia, while normal saline was used to wash the conjunctival sac. The periocular skin was disinfected with anerdian, and sterile gauze was applied.

The operation was performed under the microscope. A diameter of $3.5 \mathrm{~mm}$ trephine was used to get the imprinting of the rat cornea, and the corneal graft was punctured with the needle of a sterile syringe. The anterior chamber was successfully formed by injection of a little Aiwei viscoelastic agent into the chamber. Venus shears snipped off the graft along the imprinting mark and soaked in sterile, saline-containing cell culture dishes.

The grafts (diameter, $3.5 \mathrm{~mm}$ ) were implanted into the graft bed, and 8 - 10 stitches were interrupted with 10-0 nylon suture. The anterior chamber was formed by injecting aseptic air bubbles, and the eyelids were sutured, along with application of Tarivid® eye ointment in conjunctival sac. Normal control and allograft corneal transplantation groups were treated with $0.1 \mathrm{~mL}$ saline, while TLR2 monoclonal antibody group was given the same amount of TLR2 monoclonal antibody in place of saline. The drugs were injected under the bulbar conjunctiva daily for 5 days. Following the surgery, $0.1 \mathrm{~mL}$ of Tarivid® eye drop was applied to the conjunctival sac three times/day (morning, afternoon and evening).

\section{Parameters and scoring criteria}

Rejection index was recorded everyday with slit light microscope from first day after the operation. Corneal transparency, degree of swelling, distribution of neovascularization, and the time of corneal rejection were noted, and corneal opacity, edema and neovascularization were evaluated. When the sum of the three indices of grafts was 5 points or more, or the score of corneal opacity reached 3 , immune rejection was deemed to have occurred. Histopathological and immuno-histochemical examinations were performed on the $9^{\text {th }}$ day after the operation.

Eyeballs from 3 rats in each group were fixed with $40 \mathrm{~g} / \mathrm{L}$ paraformaldehyde solution, and dehydrated and embedded in paraffin. Serial 5$\mathrm{mm}$ sections were prepared. Then, the sections were subjected to Hematoxylin and Eosin $(\mathrm{H}$ \& E) staining. The first antibody used was TLR2 antibody, while goat-derived anti-mouse antibody was used as the second antibody. Routine Streptomyces antibiotic protein-peroxidase threestep method was applied. Following heat repair antigen, DAB color rendering and sealing, the results were observed and photographed using a confocal microscope (Nikon, Japan). In all the samples, PBS was used as the negative control, with known positive films set as the positive control. A brownish color in the cytoplasm or nucleus was considered as evidence of positively-stained cells. 


\section{RESULTS}

\section{Rejection}

Corneal edema was observed in 5 cases of allograft corneal transplantation at 1 - 4 days after operation. Corneal grafts were transparent, and neovascularization began to appear in 2 cases. At 5 to 8 days after operation, the allograft corneal transplantation group showed moderate edema, increased thickness of stroma, hidden iris vessels, and varying degrees of neovascularization in the graft bed, among which 3 cases began to develop new vessels within the grafts. In TLR2 monoclonal antibody group, corneal edema was slight, the grafts were transparent, and there were about $75 \%$ new vessels in the graft bed. At 9 - 14 days after operation, corneal new vessels in the negative control group were covered with the whole grafts; the grafts were edematous, opacity was heavy, and the pupils were not clearly visible. In the TLR2 monoclonal antibody group, corneal new vessels grew into the grafts, which were transparent and the pupils were visible. These results are shown in Figure 1.

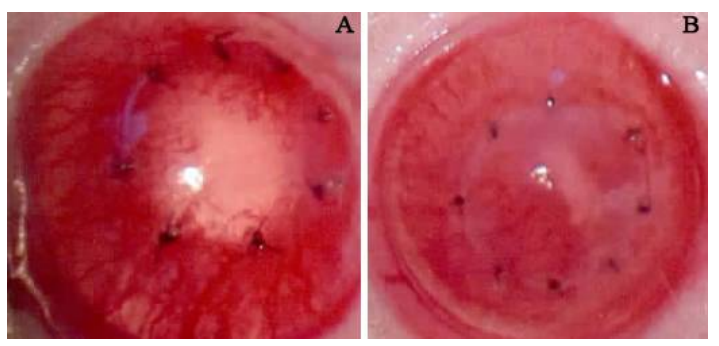

Figure 1: Corneal grafts of rats under slit lamp microscope on day 9 after operation. Note: A, negative control group; B: TLR2 monoclonal antibody group.

\section{Morphology of rat cornea}

At day 9 after operation, the corneal tissue of the normal control group was divided into 5 layers, and the structure of each layer was clear, with loose stromal layer collagen. They were arranged neatly without infiltration of inflammatory cells. In the negative control group, corneal grafts showed varying degrees of edema, thickening, disordered stromal layer structure, visible new vessels in lumen, and a large number of inflammatory cells. In TLR2 monoclonal antibody group, the corneal structure was still clear, and only a few inflammatory cells infiltrated the stromal layer. These results are displayed in Figure 2.

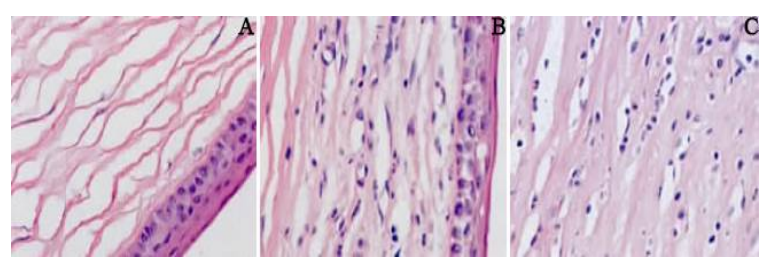

Figure 2: Rat cornea on day 9 after operation (H \& $E$ staining). Note: A, normal control group; B: TLR2 monoclonal antibody group; $\mathrm{C}$, negative control group

\section{Immuno-histochemical characteristics of TLR2 in rat corneal tissues}

At day 9 after operation, there were trace amounts of TLR2 expression in corneal epithelium of rats in the normal control group, simple control group and TLR2 monoclonal antibody group. In negative control group, the expression of TLR2 in the corneal epithelium and stromal cells was significantly increased, especially in the stromal layer (Figure 3).

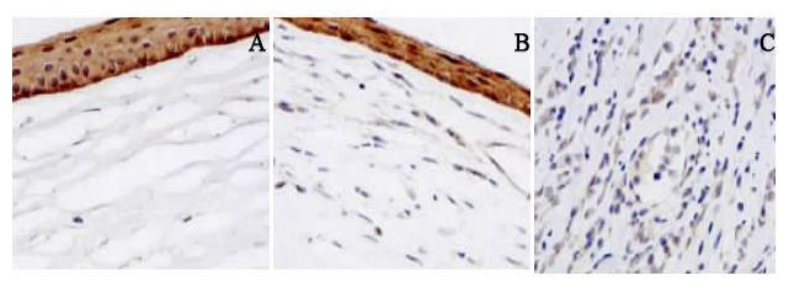

Figure 3: Immuno-histochemical staining of TLR2 in rat cornea tissues. Note: A, normal control group; B: TLR2 monoclonal antibody group; C: negative control group

\section{DISCUSSION}

Keratoplasty is the most successful organ transplantation operation, but the occurrence of postoperative immune rejection is still the main reason why the graft cannot survive after keratoplasty [9]. Toll-like receptor 2 (TLR2) is special in the TLRs family, and it is the most widely expressed protein among cloned human TLRs family members. It recognizes the most pathogenic microorganisms and their products.

The ligand specificity of TLR2 is relatively broad, mainly because when it combines with TLR1 or TLR6, it forms a heterodimer which can recognize a variety of pathogen-associated molecular patterns (PAMP). These include signal transduction of Gram positive bacteria (e.g. Staphylococcus aureus), lipoproteins, lipids, polypeptides, arabinosanthin (LAM), fungi, mycobacterium, mycoplasma lipopeptide, yeast cell wall, and peptidoglycan (PGN) [10].

Studies have shown that TLR may be involved in allograft rejection, which is dependent on TLRmediated MyD88 signal transduction [11]. The 
involvement of TLR in transplant rejection may occur through the activation of TLR and phagocytosis of TLR ligand through endogenous ligand produced by damaged cells. The activation of the secretion of pro-inflammatory cytokines by MyD88 down-transduction signal further promotes DC maturation and expression of co-stimulatory molecules and cytokines, and activation of $T$ cells, thereby mediating transplant rejection [12]. By blocking TLR2-Myd88-NF-KB and TLR2-Myd88-IRF3 pathways, TLR2 monoclonal antibody can alleviate acute rejection after transplantation and prolong the survival time of heart grafts [13].

The results of the present study revealed corneal edema in 5 cases of allograft corneal transplantation group at 1 - 4 days after operation. The corneal grafts were transparent, and neovascularization began to appear in 2 cases. At 5 to 8 days after operation, the allograft corneal transplantation group showed moderate edema, increased stromal thickness, hidden iris vessels and varying degrees of neovascularization in the graft bed, among which 3 cases began to develop new vessels within the grafts.

In TLR2 monoclonal antibody group, corneal edema was slight, the grafts were transparent, and there were $75 \%$ new vessels in the graft bed. At 9 - 14 days after operation, corneal new vessels were covered with grafts in negative control group; the grafts were edematous, with heavy opacity, and the pupils were not clearly visible. In TLR2 monoclonal antibody group, corneal new vessels grew into the grafts, which were transparent and the pupils were visible. At day 9 after operation, the corneal tissue of normal control group was divided into 5 clear layers, with loose stromal layer collagen. They were arranged neatly without inflammatory cell infiltration. In negative control group, corneal grafts showed varying degrees of edema, thickening, disordered stromal layer structure, visible new vessels in lumen, and a large number of inflammatory cells.

In TLR2 monoclonal antibody group, the corneal structure was still clear, and only a few inflammatory cells infiltrated in the stromal layer. There were trace amounts of TLR2 expression in corneal epithelium of rats in normal control group, negative control group and TLR2 monoclonal antibody group. In negative control group, the expression of TLR2 in the corneal epithelium and stromal cells was significantly increased, especially in the stromal layer.

\section{CONCLUSION}

These results indicate that TLR2 monoclonal antibody exerts remarkable and beneficial effects on neovascularization and immune rejection after corneal transplantation. Thus, it may be suitable clinically for the prevention and treatment of corneal transplantation rejection.

\section{DECLARATIONS}

\section{Conflict of Interest}

No conflict of interest associated with this work.

\section{Contribution of Authors}

This work was done by the authors named in this article and the authors accept all liability resulting from claims which relate to this article and its contents. The study was conceived and designed by Wang Ji; Yuan Jianshu, Wen Feng collected and analysed the data; Wang Ji wrote the text: All authors have read and approved the text prior to publication.

\section{REFERENCES}

1. Jia $L Z$. To intensify the activities for prevention of blindness is the social responsibility of Chinese ophthalmologists. Chin J Ophthalmol 2005; 41(1): 3-5.

2. Liang G, Zhang FJ. Advance of femtosecond small incision lenticule extraction (SMILE) in clinic application. Chin J Ophthalmol 2016; 52(1): 68-72.

3. Jerry YN. Corneal Transplantation and Immune Privilege. Int Rev Immunol 2013; 32(1): 57-67.

4. Alexandra ZC, Dipika VP, Charles NJM. A brief history of corneal transplantation: From ancient to modern. Oman J Ophthalmol 2013; 6(1): 12-17.

5. Weiyun $S$, Min $C$, Lixin $X$, Mingna $L$, Hua $G$, Ting $W$, et al. A novel cyclosporine a drug-delivery system for prevention of human corneal rejection after high-risk keratoplasty: a clinical study. Ophthalmology 2013; 120(4): 695-702.

6. Bethany MT, Daniel RG. Toll-like receptors and their role in transplantation. Front Biosci 2007; 12(12): 4221-4238.

7. Jia Z, Li F, Zeng $X, L v Y$, Zhao $S$. The effects of local administration of mesenchymal stem cells on rat corneal allograft rejection. BMC Ophthalmol 2018; 18(1): 139.

8. Lang B, Xiao HL, Feng YS, Yan YZ, Jian Y, Ming FT, et al. Blockade of toll-like receptor 2 expression and membrane translocation in rat corneal epithelial cells by glucocorticoid (TobraDex) after penetrating keratoplasty. Cornea 2011; 30(11): 1253-1259.

9. World Health Organization. Principles of laboratory animal care. WHO Chron 1985; 39: 51-56. 
10. Ting Z. Pathological and functional changes and mechanism of iris in immune rejection of corneal transplantation. Univ Qingdao 2017; 18: 33-39.

11. Takeda K, Akira S. Toll-like receptors. Curr Protoc Immunol 2007; 14: 14.

12. Akinbami A. NK Cells in Transplantation. Transplant 2017; 101(10): 2262-2264.
13. Hui XC, Lu Y, Cheng HP. Role of Toll- like receptors in organ transplantation immunity. Int J Surg 2006; 33(1): 41-43.

14. Chen GY, Brown NK, Wu W, Khedri Z, Yu H, Chen $X$, van de Vlekkert D, D'Azzo A, Zheng P, Liu Y. Broad and direct interaction between TLR and Siglec families of pattern recognition receptors and its regulation by Neu1. Elife 2017; 3(252): 04066. 